\title{
Redefining the Pulvinar Sign in Fabry Disease
}

\author{
(D). Cocozza, (D). Russo, (D) A. Pisani, (D) G. Olivo, (DE. Riccio, (D) A. Cervo, (D). Pontillo, (D). Feriozzi, (D) M. Veroux, (D). Battaglia,

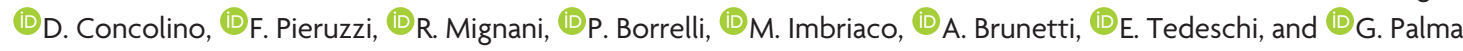

\begin{abstract}
BACKGROUND AND PURPOSE: The pulvinar sign refers to exclusive TIWI hyperintensity of the lateral pulvinar. Long considered a common sign of Fabry disease, the pulvinar sign has been reported in many pathologic conditions. The exact incidence of the pulvinar sign has never been tested in representative cohorts of patients with Fabry disease. The aim of this study was to assess the prevalence of the pulvinar sign in Fabry disease by analyzing TIWI in a large Fabry disease cohort, determining whether relaxometry changes could be detected in this region independent of the pulvinar sign positivity.
\end{abstract}

MATERIALS AND METHODS: We retrospectively analyzed brain MR imaging of 133 patients with Fabry disease recruited through specialized care clinics. A subgroup of 26 patients underwent a scan including 2 FLASH sequences for relaxometry that were compared with MRI scans of 34 healthy controls.

RESULTS: The pulvinar sign was detected in 4 of 133 patients with Fabry disease (3.0\%). These 4 subjects were all adult men (4 of 53, 7.5\% of the entire male population) with renal failure and under enzyme replacement therapy. When we tested for discrepancies between Fabry disease and healthy controls in quantitative susceptibility mapping and relaxometry maps, no significant difference emerged for any of the tested variables.

CONCLUSIONS: The pulvinar sign has a significantly lower incidence in Fabry disease than previously described. This finding, coupled with a lack of significant differences in quantitative MR imaging, allows hypothesizing that selective involvement of the pulvinar is a rare neuroradiologic sign of Fabry disease.

ABBREVIATIONS: ERT = enzyme replacement therapy; FD = Fabry disease; $\mathrm{HC}$ = healthy controls; PS = pulvinar sign; qMRI = quantitative MRI; QSM = quantitative susceptibility mapping; $\mathrm{Rl}=$ longitudinal relaxation rate; $\mathrm{R} 2=$ pure transverse relaxation rate

$\mathbf{F}_{\mathrm{b}, \mathrm{at}}$ abry disease (FD) is a rare $\mathrm{X}$-linked metabolic disorder caused by insufficient/absent lysosomal $\alpha$-galactosidase A activity. This enzymatic defect leads to pathologic storage of glycosphingolipids, especially globotriaosylceramide, occurring in all tissues

Received March 30, 2017; accepted after revision July 22.

From the Departments of Advanced Biomedical Sciences (S.C., C.R., G.O., A.C., G.P., M.I., A.B., E.T.) and Public Health (A.P., E.R.), Nephrology Unit, University "Federico II," Naples, Italy; Department of Neuroscience (G.O.), Uppsala University, Uppsala, Sweden; Nephrology and Dialysis Department (S.F.), Belcolle Hospital, Viterbo, Italy; Department of Medical and Surgical Sciences and Advanced Technologies (M.V.), University Hospital of Catania, Catania, Sicily, Italy; Department of Specialized Medicine (Y.B.), Division of Nephrology and Dialysis, St. Anna Hospital-University, Ferrara, Italy; Department of Pediatrics (D.C.), University Magna Graecia, Catanzaro, Italy; Nephrology Unit (F.P.), University of Milano-Bicocca, Milan, Italy; Nephrology and Dialysis Department (R.M.), Infermi Hospital, Rimini, Italy; IRCCS SDN (P.B.), Naples, Italy; and Institute of Biostructure and Bioimaging (G.P.), National Research Council, Naples, Italy.

Sirio Cocozza and Camilla Russo contributed equally to this work.

Enrico Tedeschi and Giuseppe Palma share senior authorship.

Please address correspondence to Camilla Russo, MD, Department of Advanced Biomedical Sciences, Università degli Studi di Napoli “Federico II," Via Pansini, 5, 80131, Naples, Italy; e-mail: camilla_russo@hotmail.it and causing multiorgan progressive dysfunction, in the kidney, heart, and central nervous system. ${ }^{1,2}$

Neurologic involvement is common in FD. ${ }^{3}$ Most prominent manifestations include cerebrovascular events, such as transient ischemic attacks and strokes, chronic cerebral vasculopathy, and vessel ectasia, especially in the posterior circulation. ${ }^{4-7}$ Such clinical manifestations translate, on brain MR imaging, in the presence of white matter hyperintensities ${ }^{3}$ and increased basilar artery diameter, ${ }^{8,9}$ both nonspecific for FD. ${ }^{10,11}$

The pulvinar sign (PS), defined as the exclusive involvement of the lateral pulvinar with symmetric hyperintensity on unenhanced T1-weighted brain MR imaging, has long been considered a common neuroradiologic sign of FD. ${ }^{8,12-16}$ Originally thought to be pathognomonic of FD, ${ }^{12,13}$ the PS has actually been reported in other conditions, such as metabolic disorders (eg, Krabbe or Tay-Sachs disease),${ }^{17,18}$ CNS infections, ${ }^{19}$ or after chemoradia-

Indicates article with supplemental on-line table.

http://dx.doi.org/10.3174/ajnr.A5420 
tion therapy ${ }^{15}$; therefore, its pathognomonic role has been largely rediscussed. ${ }^{20,21}$ In fact, the pulvinar nuclei are sensitive to metabolic disturbances, and their MR imaging appearance can be altered in a variety of conditions, especially in patients in whom an abnormal renal function can be a relevant confounding comorbidity.

Moreover, the prevalence of the PS among the FD population has been reconsidered across the years. ${ }^{14-15,22}$ To date, the exact incidence of the PS has never been tested in a large and representative cohort of patients with FD, because all studies conducted so far were limited to small samples ${ }^{13}$ or performed only on affected males. $^{12}$

In this respect, quantitative MR imaging (qMRI) and, in particular, relaxometry are likely to provide unique insight into the pathogenetic mechanisms of the PS. Indeed, a variety of microstructural conditions leading to a shorter longitudinal relaxation rate (R1) (thus, to T1WI hyperintensity) are associated with a faster free induction decay (ie, increase in $\mathrm{R} 2^{\star}$ ) and a rise of the pure transverse relaxation rate (R2). As a result, competitive roles of the longitudinal and transverse relaxation rates in MR signal generation suggest that standard evaluations of signal intensity are not the best choice for studying the incidence of PS-associated changes. A thorough relaxometry study would disentangle the contributions of several physical quantities to signal equation of conventional sequences, therefore providing a more accurate indication of the actual PS incidence.

This study has dual aims: 1) to assess the prevalence of PS in a large cohort of subjects with FD by retrospectively analyzing T1WI of 133 patients, and 2) to determine, for the first time, whether relaxometry modifications could be detected in the pulvinar, independent of the PS.

\section{MATERIALS AND METHODS Subjects}

For the retrospective analysis, in this multicenter study, we analyzed a group of 133 patients with FD (80 women, 60.1\%; mean age, $41 \pm 13.8$ years; age range, $13-73$ years) recruited in the previous 5 years through the FD specialized care clinics of 6 different hospitals. Diagnosis of FD was based on reduced plasma levels of $\alpha$ galactosidase A activity of less than average normal values, then confirmed by genotyping tests. Clinical and radiologic data were obtained in different centers as part of the clinical work-up deemed necessary for each patient, and authorization for transfer of data was formerly obtained from the local ethics committee of the coordinating center. Activity of the $\alpha$ galactosidase A enzyme was absent in 42 patients $(31.6 \%)$, while it showed a residual activity in 91 patients (68.4\%); distinct GLA gene mutations were represented, and the clinical manifestations were different, with multiple organ involvement. Finally, 85 patients were treated with enzyme replacement therapy (ERT) $(63.9 \%$, with a mean duration of $39.9 \pm 61.6$ months). Demographic and clinical information of all patients with FD in the retrospective analysis are listed in the On-line Table. For the qMRI analysis, a subgroup of 26 patients with FD (17 women, 65.4\%; mean age, $43 \pm 12.4$ years; age range, 20-68 years) underwent MR imaging at the coordinating center, along with a group of healthy controls
(HC) of comparable age and sex, without a history of neurologic, metabolic, or psychiatric disorders. Activity of the $\alpha$ galactosidase A enzyme was absent in 7 patients (26.9\%), while it had residual activity in 19 patients $(73.1 \%)$, with 25 patients treated with ERT (96.2\%; mean therapy duration, $43.8 \pm 51.2$ months). Demographic and clinical information of the qMRI subgroup of patients with FD are also listed in the On-line Table.

This study was performed in accordance with the ethical standards of the Declaration of Helsinki, and written informed consent for the qMRI analysis was preliminarily obtained from the 26 patients with FD and the HC.

Inclusion criteria for patients with FD were the following: genetically proved $\mathrm{FD}$; availability of documentation of brain MR images, including at least 1 spin-echo T1WI sequence independent of the orientations with a section thickness $<4 \mathrm{~mm}$; and signed informed consent for participation in the study for subjects undergoing the qMRI analysis. Both adult male and female subjects were approached to participate, without age limitations. Diagnosis of FD was confirmed by biochemical or genetic testing. Indications for brain MR imaging varied between and within centers, including, but not limited to, routine MR imaging, headache, transient neurologic symptoms, and evaluation of an acute cerebrovascular accident. Exclusion criteria for all subjects were evidence of hypo- or hypercalcemia or the presence of any other comorbidity that could have biased the neuroradiologic examination.

From each specialized care center participating in this study, demographic and clinical variables were extracted by a clinician experienced in FD from the medical records or obtained in direct interviews and recorded. These included age recorded at the time of the brain MR imaging, sex, hypertension, diabetes mellitus, cardiac arrhythmia, left ventricular hypertrophy, renal failure (considered present when the estimated glomerular filtration rate of the patient was $<90 \mathrm{~mL} / \mathrm{min}$ ), proteinuria (considered present when the patient scored a value $>150 \mathrm{mg} / 24$ hours), the presence of neurologic symptoms (including stroke, cephalalgia, acroparesthesia, and so forth), gastrointestinal involvement, renal or cardiac transplant, current use of tobacco or alcohol, as well as treatment with ERT. About $63 \%$ of patients with renal failure showed metabolic alterations of parathyroid hormone serum levels attributable to secondary hyperparathyroidism, with no significant derangements of serum calcium levels. Renal function was expressed as the estimated glomerular filtration rate, calculated with the Chronic Kidney Disease Epidemiology Collaboration equation. ${ }^{23}$ Other laboratory values were measured using standard hospital laboratory techniques. Activity of the $\alpha$ galactosidase A enzyme was reported as "absent" (when showing $<1 \%$ of normal values) or "residual" (for enzyme activity ranging from $1 \%$ to $5 \%$ of normal values) because of the different measurement methods used in the recruiting centers (plasma, leukocytes, and so forth).

Each center received a dedicated case report form in which demographic and clinical information were recorded, and then forms were entered into a central data base at the coordinating center according to Good Clinical Practice guidelines.

AJNR Am J Neuroradiol 38:2264-69 Dec 2017 www.ajnr.org 

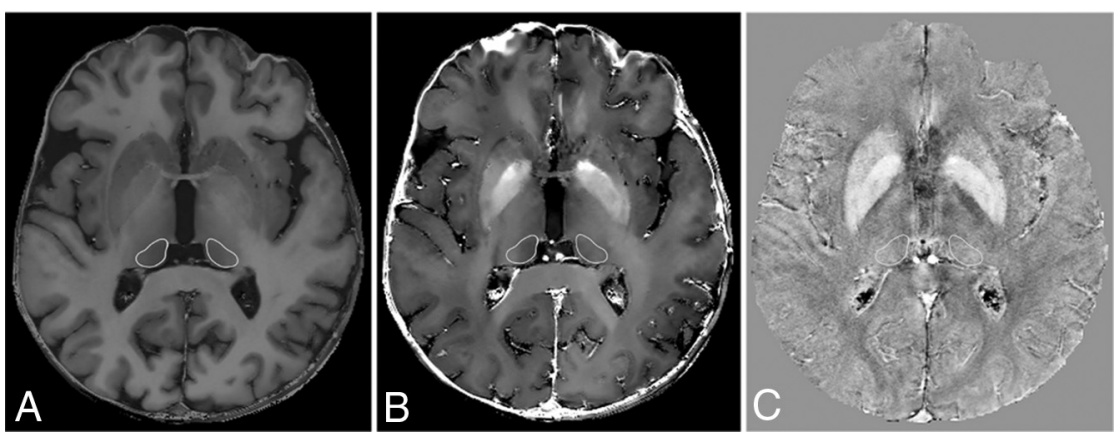

FIG 1. RT $(A), R 2^{*}(B)$, and QSM (C) maps showing the 2 hand-drawn irregular bilateral ROIs placed in consensus by 2 experienced neuroradiologists on the axial section of the gradient recalledecho images with the best representation of the pulvinar nuclei.

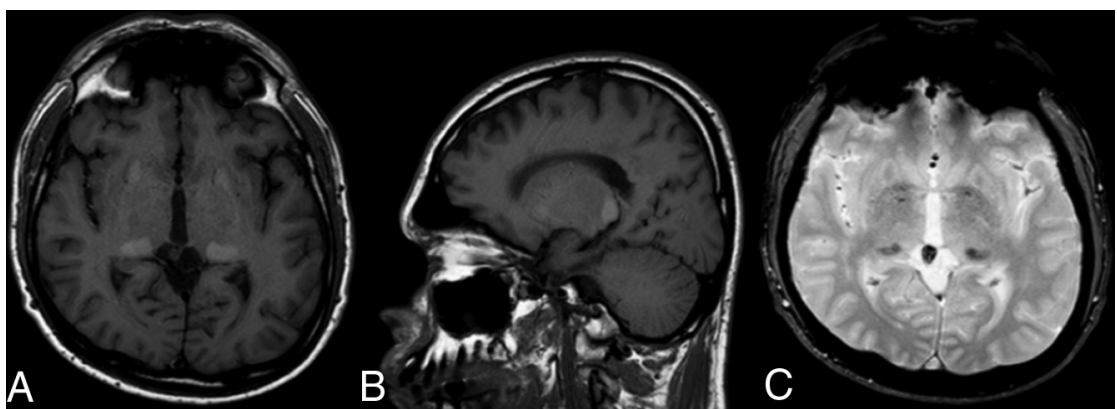

FIG 2. Unenhanced TIWI axial $(A)$ and sagittal $(B)$ images showing bilateral, symmetric, and wellcircumscribed hyperintensity of the pulvinar nuclei in a 47-year-old male patient with FD (A). Axial $\mathrm{T}^{*}$ gradient echo image $(C)$ shows bilateral and symmetric hypointensity corresponding exactly to the $\mathrm{Tl}$ hyperintensity, supporting the current hypothesis of focal calcium deposition in both pulvinar nuclei.

\section{qMRI Data Acquisition}

All studies for the qMRI analysis were performed on the same 3T MR imaging scanner (Magnetom Trio; Siemens, Erlangen, Germany). The acquisition protocol included a dual-echo spoiled gradient-echo (FLASH) sequence $\left(\mathrm{TR}=32 \mathrm{~ms}, \mathrm{TE}_{1}=7.38 \mathrm{~ms}, \mathrm{TE}_{2}=22.14 \mathrm{~ms}\right.$, flip angle $=20^{\circ}$ ) and a single-echo FLASH sequence ( $\mathrm{TR}=16 \mathrm{~ms}$, $\mathrm{TE}=7.38 \mathrm{~ms}$, flip angle $=2^{\circ}$ ). Both sequences were acquired on an FOV of $230 \times 194 \times 160 \mathrm{~mm}^{3}$, with a voxel size of $0.5 \times 0.5 \times 1.0$ $\mathrm{mm}^{3}$ and a receiver bandwidth of $100 \mathrm{~Hz} /$ pixel.

The acquired data were saved as complex datasets reconstructed in magnitude and phase representation for further analyses.

\section{MR Imaging Data Analysis}

For the retrospective analysis, MR imaging brain scans were visually assessed by 2 neuroradiologists in consensus, who rated the presence of a clear unilateral or bilateral T1WI hyperintensity at the level of the posterior thalami.

For the qMRI analysis, all magnitude datasets underwent a preliminary multispectral denoising as described in Borrelli et al, ${ }^{24}$ using a multi-graphics processing unit implementation of the non-local means algorithm previously introduced. ${ }^{25}$ Quantitative susceptibility mapping (QSM) and R2* maps were derived from the dual-echo FLASH. ${ }^{26}$ Conversely, given the signal equation of FLASH with $\mathrm{FA}=\theta$

$$
S=K|\sin \theta| \frac{1-E_{1}}{1-E_{1} \cos \theta} e^{-T_{\mathrm{E}} R_{2}^{*}},
$$

\section{Statistical Analysis} analysis in consensus.

\section{RESULTS} PS is shown in Fig 2.

$$
E_{1}=e^{-T_{\mathrm{R}} R_{1}},
$$

it can be shown that assuming a $\mathrm{T} 1>3$ $\mathrm{ms}$, the actual settings of the acquisition parameters $\left(\mathrm{TR}=16 \mathrm{~ms}, \theta_{1}=2^{\circ}, \theta_{2}=\right.$ $20^{\circ}$ ) allow R1 computation as

$$
R_{1}=\frac{1}{T_{\mathrm{R}}} \log \frac{R+\sqrt{R^{2}+4 C(r-k)}}{2 C},
$$

where

$$
\begin{gathered}
r=\frac{S_{1}}{S_{2}}, \\
k=\frac{\left|\sin \theta_{1}\right|}{\left|\sin \theta_{2}\right|}, \\
R=r\left(1-\cos \theta_{1}\right),
\end{gathered}
$$

and

$$
C=r \cos \theta_{1}-k \cos \theta_{2} .
$$

For each study, 2 irregular bilateral ROIs were hand-drawn in consensus by 2 experienced neuroradiologists on axial sections of the gradient recalled-echo images with the best representation of pulvinar nuclei (Fig 1), according to a human anatomy atlas. ${ }^{27}$ Mean R1, R2*, and QSM values were automatically obtained from the corresponding maps.

Statistical analyses were performed with the Statistical Package for the Social Sciences (Version 17.0; IBM, Armonk, New York). An independent 2-sample $t$ test was used for comparing ages and mean values obtained from qMRI analysis, while a $\chi^{2}$ test was used to determine differences in terms of sex. A $P=.05$ indicated a statistically significant difference. Finally, no Cohen coefficient was needed to evaluate the performance of the evaluation because the 2 neuroradiologists performed the

For the retrospective analysis, a clear T1WI hyperintensity of the pulvinar was detected in 4 of 133 patients with FD (3.0\%). All 4 patients with positive PSs underwent MR imaging examinations in 2 of the 6 hospitals involved; they all were adult male subjects with an age ranging from 38 to 59 years (mean, $46.5 \pm 9.4$ years) under ERT and showing signs of renal failure. An example of the

For the qMRI analysis, the FD and HC groups were comparable for age and sex. No subject with a positive PS was found in the qMRI subgroup. When we tested for possible differences between these 2 clusters in relaxometry and QSM maps in the FD and HC groups, no significant differences emerged. A complete list of the results obtained by relaxometry and susceptibility analysis, along with the comparison of $P$ values, is shown in the Table. 
Results of the relaxometry analysis ${ }^{\mathrm{a}}$

\begin{tabular}{lccc}
\hline & HC & FD & $\boldsymbol{P}$ Value \\
\hline Right pulvinar area & $49 \pm 7$ & $46 \pm 6$ & .14 \\
Left pulvinar area & $53 \pm 7$ & $51 \pm 10$ & .41 \\
Right pulvinar R1 & $1.31 \pm 0.11$ & $1.33 \pm 0.09$ & .45 \\
Left pulvinar R1 & $1.41 \pm 0.14$ & $1.44 \pm 0.12$ & .32 \\
Right pulvinar R2* & $19.9 \pm 1.7$ & $20.6 \pm 3.4$ & .35 \\
Left pulvinar R2* $^{*}$ & $19.9 \pm 1.9$ & $20.3 \pm 2.8$ & .55 \\
Right pulvinar QSM & $40 \pm 19$ & $41 \pm 19$ & .75 \\
Left pulvinar QSM & $38 \pm 17$ & $35 \pm 18$ & .56
\end{tabular}

Note:- R1 indicates the longitudinal relaxation rate $(1 / T)$, expressed in second ${ }^{-1} ; R^{*}$, transverse relaxation rate $\left(1 / \mathrm{T} 2^{*}\right)$, expressed as second ${ }^{-1}$.

${ }^{a}$ QSM is expressed in parts per billion. Areas are expressed in square millimeters. $P$ values refer to an independent 2-sample $t$ test, with a significance level of $P=.05$.

\section{DISCUSSION}

Our findings support the hypothesis that the true incidence of the PS is considerably lower than previously reported. No significant qMRI differences emerged when testing for possible alterations in subjects without positive PSs, allowing us to speculate that this sign is poorly sensitive for FD.

Exclusive involvement of the lateral pulvinar was first observed by 2 separate groups. ${ }^{12,13}$ Since then, this sign has been considered as distinctive of FD. Independent from its diagnostic value, the exact incidence of the PS has changed across time, ${ }^{14,15}$ with a clear downward trend going from $70 \%{ }^{13}$ of earlier studies (though reported in a very small group of patients without any ascertainment data) to complete absence in recent works. ${ }^{20} \mathrm{How}-$ ever, the real incidence of the PS has never been tested in an ample-sized group of patients with FD. Here we expand this knowledge by performing a retrospective analysis of a large and representative population of patients with FD. We observed a prevalence of the PS in FD of 3.0\%, significantly lower than previously reported..$^{8,12-16}$ A possible explanation for this discrepancy could reflect the relatively small sample size of some of the previous studies. ${ }^{15}$

Nevertheless, when considering the study by Moore et al, ${ }^{12}$ which included a number of subjects comparable with ours, a higher incidence of the PS was observed. However, the abovementioned study included only male patients with $\mathrm{FD},{ }^{12}$ in whom the PS is known to be more frequent, ${ }^{21}$ and this could have somehow biased the real prevalence of the PS in the global FD population. Supporting this speculation, we were able to replicate such findings when we selected only males with renal function impairment, ${ }^{28}$ showing a PS prevalence similar to the one reported in Moore et al (4/25, 16.0\%).

Another explanation of the previously reported high incidence of a positive PS in patients with FD may be related to possible gadolinium deposition in this structure. Indeed, recent evidence suggests that in subjects with normal renal function receiving multiple contrast administrations, the dentate nuclei and other deep gray matter structures, including the globus pallidus and the pulvinar, could accumulate gadolinium, leading to changes detectable on both conventional and quantitative imaging. ${ }^{29-31}$ It may, therefore, be hypothesized that the reduced glomerular filtration rate in patients with FD could have influenced the gadolinium clearance, leading to its accumulation in the pulvinar nuclei with subsequent T1WI shortening. However, in patients with FD, T1WI hyperintensity was not observed in the dentate nuclei, which is the preferred site of gadolinium accumulation, and contrast media are rarely administered in these patients, due to their usually impaired renal function. Even though this evidence mitigates the hypothesis of gadolinium accumulation in patients with FD, further properly designed studies may better address the possible relationships between impaired renal function and metal buildup in the pulvinar.

In line with literature data, ${ }^{21}$ no affected females with the PS were found, considered a rare event ${ }^{16}$ due to partial/residual enzyme activity. The mean age of our PS-positive patients was 46.5 years, confirming the current hypothesis that the PS could be the neuroradiologic epiphenomenon of a long-term accumulation, whose manifestations only appear progressively. ${ }^{15}$

Other literature data indicate the poor prognostic value of the PS. Indeed, it has been demonstrated that the PS is somehow independent of major cerebrovascular events, which may occur at any stage of disease. ${ }^{21}$ Furthermore, no changes in the pulvinar nuclei occurred after long-term ERT, and no association between the PS and a specific genotype has been demonstrated. ${ }^{15}$

Aside from the incidence of the PS, we aimed to evaluate whether possible changes in susceptibility maps could occur in the pulvinar of patients with FD.

It is widely accepted that the pulvinar is a sensitive region where alterations could develop, independent from the cause. Selective modifications of pulvinar nuclei have been described in other pathologic conditions, such as Wernicke encephalopathy ${ }^{32,33}$ and Creutzfeldt-Jakob ${ }^{34,35}$ and Tay-Sachs diseases. ${ }^{17}$ In particular, T1WI hyperintensity in the pulvinar has also been described in patients who received chemotherapy/radiation therapy, in patients with neurofibromatosis type 1, and in subjects with Fahr disease or disturbances of the calcium-phosphorus metabolism or even laminar necrosis. ${ }^{36-38}$

To date, the real pathogenesis of the PS in FD is still unclear. The most reliable hypothesis is the development of subtle dystrophic calcification, confirmed by CT brain scans, probably related to chronic hypoperfusion secondary to microvascular alterations. ${ }^{6,14,39-41}$ These alterations have been reported not only in the pulvinar but also in other deep gray nuclei such as the globus pallidus, even if in a lower percentage of cases. ${ }^{12-14}$

Quantitative MR imaging can allow early detection of changes not appreciable on conventional MR images ${ }^{42}$ possibly providing additional information in understanding PS physiopathology. However, we found no differences in the pulvinar in any of the examined qMRI maps of patients with FD compared with HC. This absence of significant differences, however, should not lead to the superficial deduction that the PS is not linked to abnormal mineral deposition. Unfortunately, in our qMRI FD subgroup, no subjects with clear PSs were present because of the low incidence of this sign. On the other hand, in patients positive for the PS, a clinical T2*WI FLASH sequence was available and showed clear hypointensity corresponding to T1WI hyperintensity, corroborating the current hypothesis of calcium deposition in the posterior thalami when the PS is present. A possible explanation for the absence of sensitive mineralization in the PS-negative group might be related to the ongoing therapy, since the FD subgroup with available qMRI sequences almost entirely consisted of subjects under ERT (25/26 patients). Indeed, due to the current 
pathogenetic assumption that microvascular changes are responsible for PS development, it can be hypothesized that early diagnosis of FD, coupled with the availability of effective treatment options, could have directly or indirectly improved local microvascular homeostasis. Nonetheless, this speculation should be corroborated by further longitudinal evaluations of qMRI, to clarify the relationship between these measures and ERT.

\section{CONCLUSIONS}

We showed a significantly lower incidence (only 3\%) of the PS in FD compared with what has been previously described, coupled with no significant qMRI differences in these regions between those with FD and HC. Our results, taken together, allow hypothesizing that selective pulvinar involvement with well-circumscribed T1-hyperintensity, though easy to identify, is a rare neuroradiologic sign whose presence is detectable in only a few exceptional cases of FD. Moreover, because this finding has also been reported in other conditions of metabolic dysfunction, it should not be considered specifically related to globotriaosylceramide accumulation and its role as a sensitive sign of this disease should be reconsidered.

Disclosures: Sirio Cocozza—UNRELATED: Payment for Lectures Including Service on Speakers Bureaus: Genzyme; Travel/Accommodations/Meeting Expenses unrelated to Activities Listed: Genzyme. Antoni Pisani-UNRELATED: Consultancy: Genzyme, Shire, Amicus; Payment for Lectures Including Service on Speakers Bureaus: Genzyme, Shire, Amicus; Travel/Accommodations/Meeting Expenses unrelated to Activities Listed: Genzyme, Shire, Amicus. Eleonora Riccio-UNRELATED: Payment for Lectures Including Service on Speakers Bureaus: Genzyme, Shire; Travel/Accommodations/Meeting Expenses unrelated to Activities Listed: Genzyme, Shire. Daniela Concolino_-UNRELATED: Payment for Lectures Including Service on Speakers Bureaus: Shire; Travel/Accommodations/Meeting Expenses unrelated to Activities Listed: Shire. Federico Pieruzzi-UNRELATED: Payment for Lectures Including Service on Speakers Bureaus: Genzyme, Shire, Amicus; Travel/Accommodations/ Meeting Expenses unrelated to Activities Listed: Genzyme, Shire, Amicus. Sandro Feriozzi-UNRELATED: Payment for Lectures Including Service on Speakers Bureaus: Genzyme, Shire; Travel/Accommodations/Meeting Expenses unrelated to Activities Listed: Genzyme, Shire. Renzo Mignani-UNRELATED: Payment for Lectures Including Service on Speakers Bureaus: Genzyme, Shire; Travel/Accommodations/Meeting Expenses unrelated to Activities Listed: Genzyme, Shire. Massimiliano Veroux-UNRELATED: Payment for Lectures Including Service on Speakers Bureaus: Genzyme, Shire; Travel/Accommodations/Meeting Expenses unrelated to Activities Listed: Genzyme, Shire. Yuri Battaglia-UNRELATED: Payment for Lectures Including Service on Speakers Bureaus: Genzyme; Travel/Accommodations/ Meeting Expenses unrelated to Activities Listed: Genzyme.

\section{REFERENCES}

1. Eng CM, Germain DP, Banikazemi M, et al. Fabry disease: guidelines for the evaluation and management of multi-organ system involvement. Genet Med 2006;8:539-48 CrossRef Medline

2. Germain DP. Fabry disease. Orphanet J Rare Dis 2010;5:30 CrossRef Medline

3. Buechner S, Moretti M, Burlina AP, et al. Central nervous system involvement in Anderson-Fabry disease: a clinical and MRI retrospective study. J Neurol Neurosurg Psychiatry 2008;79:1249-54 CrossRef Medline

4. Kaye EM, Kolodny EH, Logigian EL, et al. Nervous system involvement in Fabry's disease: clinicopathological and biochemical correlation. Ann Neurol 1988;23:505-09 CrossRef Medline

5. Mitsias P, Levine SR. Cerebrovascular complications of Fabry's disease. Ann Neurol 1996;40:8-17 CrossRef Medline

6. Moore DF, Kaneski CR, Askari H, et al. The cerebral vasculopathy of Fabry disease. J Neurol Sci 2007;257:258-63 CrossRef Medline

7. Fellgiebel A, Keller I, Marin D, et al. Diagnostic utility of different MRI and MR angiography measures in Fabry disease. Neurology 2009;72:63-68 CrossRef Medline
8. Fellgiebel A, Keller I, Martus $\mathrm{P}$, et al. Basilar artery diameter is a potential screening tool for Fabry disease in young stroke patients. Cerebrovasc Dis 2011;31:294-99 CrossRef Medline

9. Manara R, Carlier RY, Righetto S, et al. Basilar artery changes in Fabry disease. AJNR Am J Neuroradiol 2017;38:531-36 CrossRef Medline

10. Putaala J, Kurkinen M, Tarvos V, et al. Silent brain infarcts and leukoaraiosis in young adults with first-ever ischemic stroke. $\mathrm{Neu}$ rology 2009;72:1823-29 CrossRef Medline

11. Uçeyler N, Homola GA, Guerrero González H, et al. Increased arterial diameters in the posterior cerebral circulation in men with Fabry disease. PLoS One 2014;9:e87054 CrossRef Medline

12. Moore DF, Ye F, Schiffmann R, et al. Increased signal intensity in the pulvinar on T1-weighted images: a pathognomonic MR imaging sign of Fabry disease. AJNR Am J Neuroradiol 2003;24:1096-101 Medline

13. Takanashi J, Barkovich AJ, Dillon WP, et al. T1 hyperintensity in the pulvinar: key imaging feature for diagnosis of Fabry disease. AJNR Am J Neuroradiol 2003;24:916-21 Medline

14. Fellgiebel A, Mazanek M, Whybra C, et al. Pattern of microstructural brain tissue alterations in Fabry disease: a diffusion-tensor imaging study. J Neurol 2006;253:780-87 CrossRef Medline

15. Burlina AP, Manara R, Caillaud C, et al. The pulvinar sign: frequency and clinical correlations in Fabry disease. J Neurol 2008;255:738-44 CrossRef Medline

16. Burlina AP, Politei J, Cinque S, et al. The pulvinar sign in Fabry patients: the first report in female patients. J Neurol 2012;259: 1227-28 CrossRef Medline

17. Mugikura S, Takahashi S, Higano S, et al. MR findings in Tay-Sachs disease. J Comput Assist Tomogr 1996;20:551-55 CrossRef Medline

18. Kendall BE. Disorders of lysosomes, peroxisomes, and mitochondria. AJNR Am J Neuroradiol 1992;13:621-53 Medline

19. Sahraian MA, Motamedi M, Azimi AR, et al. Bilateral pulvinar thalamic calcification in a patient with chronic cryptococcal meningitis. Eur J Neurol 2007;14:e1-2 Medline

20. Fazekas F, Enzinger C, Schmidt R, et al; SIFAP 1 Investigators. Brain magnetic resonance imaging findings fail to suspect Fabry disease in young patients with an acute cerebrovascular event. Stroke 2015; 46:1548-53 CrossRef Medline

21. Kolodny E, Fellgiebel A, Hilz MJ, et al. Cerebrovascular involvement in Fabry disease: current status of knowledge. Stroke 2015;46: 302-13 CrossRef Medline

22. Lee HJ, Hsu TR, Hung SC, et al. A comparison of central nervous system involvement in patients with classical Fabry disease or the later-onset subtype with the IVS4+919G>A mutation. BMC Neurol 2017;17:25 CrossRef Medline

23. Vučić Lovrenčić M, Radišić Biljak V, Božičević S, et al. Estimating glomerular filtration rate (GFR) in diabetes: the performance of MDRD and CKD-EPI equations in patients with various degrees of albuminuria. Clin Biochem 2012;45:1694-96 CrossRef Medline

24. Borrelli P, Palma G, Tedeschi E, et al. Improving signal-to-noise ratio in susceptibility weighted imaging: a novel multicomponent non-local approach. PLoS One 2015;10:e126835 CrossRef Medline

25. Palma G, Comerci M, Alfano B, et al. 3D non-local means denoising via multi-GPU. Fed Conf Comput Sci 2013:495-98

26. Palma G, Tedeschi E, Borrelli P, et al. A novel multiparametric approach to 3D quantitative MRI of the brain. PLoS One 2015;10: e0134963 CrossRef Medline

27. Schaltenbrand G, Wahren W. Atlas for Stereotaxy of the Human Brain. 2nd ed. Stuttgart: Thieme; 1977

28. Branton M, Schiffmann R, Kopp JB. Natural history and treatment of renal involvement in Fabry disease. J Am Soc Nephrol 2002; 13(suppl 2):S139-43 Medline

29. Kanda T, Ishii K, Kawaguchi H, et al. High signal intensity in the dentate nucleus and globus pallidus on unenhanced T1-weighted MR images: relationship with increasing cumulative dose of a gadolinium-based contrast material. Radiology 2014;270:834-41 CrossRef Medline 
30. Ramalho J, Semelka RC, Ramalho M, et al. Gadolinium-based contrast agent accumulation and toxicity: an update. AJNR Am J Neuroradiol 2016;37:1192-98 CrossRef Medline

31. Tedeschi E, Palma G, Canna A, et al. In vivo dentate nucleus MRI relaxometry correlates with previous administration of gadolinium-based contrast agents. Eur Radiol 2016;26:4577-84 CrossRef Medline

32. Torvik A, Lindboe CF, Rogde S. Brain lesions in alcoholics: a neuropathological study with clinical correlations. J Neurol Sci 1982;56: 233-48 CrossRef Medline

33. Antunez E, Estruch R, Cardenal C, et al. Usefulness of CT and MR imaging in the diagnosis of acute Wernicke's encephalopathy. AJR Am J Roentgenol 1998;171:1131-37 CrossRef Medline

34. Zeidler M, Sellar RJ, Collie DA, et al. The pulvinar sign on magnetic resonance imaging in variant Creutzfeldt-Jakob disease. Lancet 2000;355:1412-18 CrossRef Medline

35. Keohane C. Pulvinar sign on MRI images in variant CreutzfeldtJakob disease. Lancet 2000;355:1384 CrossRef Medline

36. Ginat DT, Meyers SP. Intracranial lesions with high signal intensity on T1-weighted MR images: differential diagnosis. Radiographics 2012;32:499-516 CrossRef Medline

37. Renard D, Castelnovo G, Campello C, et al. Thalamic lesions: a radiological review. Behav Neurol 2014;2014:154631 CrossRef Medline

38. Zaitout Z, Romanowski C, Karunasaagarar K, et al. A review of pathologies associated with high T1W signal intensity in the basal ganglia on magnetic resonance imaging. Pol J Radiol 2014;79: 126-30 CrossRef Medline

39. Moore DF, Altarescu G, Barker WC, et al. White matter lesions in Fabry disease occur in 'prior' selectively hypometabolic and hyperperfused brain regions. Brain Res Bull 2003;62:231-40 CrossRef Medline

40. Igarashi T, Sakuraba H, Suzuki Y. Activation of platelet function in Fabry's disease. Am J Hematol 1986;22:63-67 CrossRef Medline

41. DeGraba T, Azhar S, Dignat-George F, et al. Profile of endothelial and leukocyte activation in Fabry patients. Ann Neurol 2000;47: 229-33 Medline

42. Deoni SC. Quantitative relaxometry of the brain. Top Magn Reson Imaging 2010;21:101-13 CrossRef Medline 